\title{
Eosinophilic Esophagitis - Treatment of Eosinophilic Esophagitis with Drugs: Corticosteroids
}

\author{
Ulrike von Arnim Peter Malfertheiner \\ Department of Gastroenterology, Hepatology and Infectious Diseases, Otto von Guericke University, \\ Magdeburg, Germany
}

\begin{abstract}
Key Words
Eosinophilic esophagitis · Treatment · Topical

corticosteroids · Fluticasone $\cdot$ Budesonide
\end{abstract}

\begin{abstract}
Eosinophilic esophagitis (EoE) is defined as a chronic immune/antigen-mediated esophageal disease characterized by clinical symptoms of esophageal dysfunction and histologically by eosinophil-predominant infiltration of the esophagus mucosa. Treatment of EoE should therefore lead to improvement of clinical symptoms and a histological decrease of eosinophilic inflammation. Topical corticosteroids (tCS; fluticasone, budesonide) have been demonstrated to be effective in treating EoE and therefore represent first-line therapy. To date, there is no approved therapy in the world for EoE. This is the reason why EoE patients are treated with medication such as inhalers or aqueous nebulizer solutions, which have to be swallowed. After administration, patients are not allowed to eat or drink for $45 \mathrm{~min}$ in order for the esophagus to become well coated so that the topical antiinflammatory effect is maximized. For adults there are different dose ranges: fluticasone propionate $440-880 \mu \mathrm{g} /$ day twice daily, budesonide $2 \mathrm{mg} /$ day divided dose for inducing remission over a period of 4 to 8 weeks. Since EoE is a chronic disease known to relapse after termination of medication, maintenance therapy seems to be valuable, but there is no evidence from controlled studies with long-term follow-up.
\end{abstract}

In several randomized trials, both tCS have been able to show a good safety profile. Candida esophagitis and oral candidiasis present the most common side effects of topical steroid therapy. At the moment, no data about tCS and long-term safety in EoE are available. @ 2014 S. Karger AG, Basel

\section{Introduction}

Eosinophilic esophagitis (EoE) is a chronic immune/ antigen-mediated esophageal disease characterized clinically by symptoms related to esophageal dysfunction and histologically by eosinophil-predominant inflammation [1]. EoE has become increasingly recognized over the last decade both in children and adults. Clinical and pathophysiologic characteristics of EoE resemble allergic asthma, which is why EoE is referred to as 'asthma of the esophagus' [2].

The definition of EoE includes clinical symptoms of esophageal dysfunction as well as eosinophilic infiltration of the mucosa of the esophagus. These two main characteristics of EoE dominate the current discussion of treatment endpoints. Whether treatment of EoE should focus on clinical symptom relief and/or achieving histologic remission is under debate. The issue of therapeutic endpoints is complicated by the heterogeneity to determine a complete histological or clinical response. Depending on the study de-

\section{KARGER}

E-Mail karger@karger.com

www.karger.com/ddi
(C) 2014 S. Karger AG, Basel

0257-2753/14/0322-0126\$39.50/0
Dr. Ulrike von Arnim

Gastroenterologie/Hepatologie, Universitätsklinikum

Otto-von-Guericke-Universität, Leipziger Strasse 44

DE-39102 Magdeburg (Germany)

E-Mail Ulrike.vonarnim@med.ovgu.de 
sign, histological responses vary from $0-1$ eosinophils/ high-power field [3, 4] to 0-6 eosinophils/high-power field [5], or a decrease of eosinophils by $90 \%$ [6]. Symptoms in EoE patients are nonspecific and can be modified by eating habits (e.g. diet), which makes it difficult to measure and evaluate for defining symptom endpoints. Results of randomized trials have demonstrated such conflicting circumstances. An overall correlation of histological and symptomatic response to treatment was demonstrated in several randomized trials [3-5, 7]. Nevertheless, in other EoE treatment studies, histologic response did not lead to a significant improvement of symptoms $[6,8,9]$. These conflicting results and interpretations underline the need for defining evidence-based therapeutic endpoints in EoE.

\section{Why Should We Treat EoE?}

\section{Quality of Life}

Having a chronic disease often changes a patient's quality of life and social experiences. Taft et al. [10] performed a study about health-related quality of life and the psychosocial impact of EoE in adults. They demonstrated that EoE has a substantial impact on several psychosocial domains which are not adequately addressed by focusing assessment only on dysphagia. Another study analyzed the psychosocial impact of EoE in children and their families. Pediatric quality of life scores were worse in children with active EoE and dietary restrictions [11]. Both studies demonstrate that EoE patients suffer from the impact on eating, although EoE patients are able to integrate their swallowing difficulties in daily life.

\section{Prevention of Food Impaction}

Food impaction in EoE patients is common, with frequencies ranging from 25 to $100 \%$ in previous studies, depending on the specific population [12]. Prospective studies have reported that $50-55 \%$ of patients presenting with food bolus obstruction fulfilled the histological criteria for EoE [13, 14]. Endoscopic removal of impacted bolus is the therapy of choice in this case and should be performed using flexible endoscopy instead of rigid endoscopy. In a retrospective study from Switzerland [15] studying procedure-induced complications in EoE patients requiring flexible or rigid endoscopic bolus removal, it was demonstrated that such complications are likely to be increased especially when using a rigid endoscopic regime (transmural perforation of esophagus: approx. $20 \%$ ). Bolus removal by rigid endoscopy represents a risky procedure and should be avoided in EoE patients.

\section{Prevention of Remodeling of the Esophagus}

It is thought that long-lasting esophageal inflammation leads to remodeling of the esophageal wall. Based on imaging of airway remodeling in patients with bronchial asthma, a sequela might be a fragile and vulnerable esophageal mucosa. Aceves et al. [16] performed a quantitative immunohistochemical study to analyze remodeling changes in biopsies of the esophagus from children with EoE, gastroesophageal reflux disease, and controls. Esophageal biopsies in EoE patients demonstrated increased levels of subepithelial fibrosis in comparison to gastroesophageal reflux disease patients and controls.

Remodeling of the esophagus leads to a loss of elasticity and narrowing of the esophageal lumen [17]. From studies in EoE patients under treatment with topical corticosteroids (tCS), it could be shown that tissue remodeling can be reversed with anti-inflammatory therapy $[3,18]$.

\section{How Should We Treat EoE?}

\section{Treatment of EoE with CS}

Systemic (oral) CS were one of the first treatment options shown to be effective in pediatric EoE patients [19]. Clinical and histological improvement was noted in up to $95 \%$ of EoE patients. Discontinuation of therapy led to a recurrence of symptoms in $90 \%$ of the patients. Prolonged use of systemic CS is associated with well-known and significant secondary effects, which explains why long-term use is not recommended. Systemic CS should be reserved for emergency cases such as in patients with dysphagia requiring hospitalization or patients with weight loss and/or dehydration due to swallowing problems. Topical steroid resistance could also be an indication for the use of a systemic CS therapy.

Because of the limited use of systemic CS, several randomized controlled studies followed using tCS in pediatric and adolescent/adult EoE patients. Since there is no approved treatment for EoE and given their substantially better safety profile, topical steroids delivered to the esophagus have become the mainstay of pharmacotherapy for EoE patients.

\section{Fluticasone}

In pediatric EoE trials comparing fluticasone (FLU) to prednisone [7] and placebo [4], an approximately 50\% complete response and 95\% partial response could be demonstrated using doses of $220-440 \mu \mathrm{g}$ for up to 3 months. In a placebo-controlled trial in adult EoE patients, FLU could induce histological remission in $62 \%$, but symptom improvement in the FLU group was not superior to placebo [6]. 
Another trial compared FLU and esomeprazole for inducing a histological and symptomatic response in EoE patients over a period of 8 weeks. There was no difference in the degree of improvement in dysphagia or eosinophil infiltration in patients treated with either FLU or esomeprazole [8]. However, this study had certain limitations such as small sample size combined with a high drop-out rate and probably a high proportion of proton pump inhibitor-responsive eosinophilic eosinophilia.

FLU is delivered to the esophagus via a pressurized metered dose inhaler that is activated into the mouth (without inhaling and without a spacer) and then finally swallowed. Patients should be advised not to eat, drink, or rinse their mouth for 20-30 min after receiving FLU.

\section{Budesonide}

In several randomized trials, budesonide (BUD) has been shown to be an effective therapy for active EoE. A retrospective analysis of children suffering from EoE and being treated with an oral viscous BUD suspension revealed a significant reduction of symptoms ( $80 \%$ responders) and histological remission [20]. Children with EoE showed significant improvement in symptoms and esophageal eosinophilia when treated with an oral viscous BUD formulation in comparison to placebo [5]. In adult EoE, treatment with nebulized oral BUD (1 mg twice daily for 15 days) was highly effective in inducing clinical and histological remission compared to placebo [3]. Another study examined two topical BUD formulations (nebulized vs. oral viscous) in a randomized openlabel setting in adult EoE [9]. Viscous BUD was more effective in inducing histological remission than nebulized swallowed BUD. The superiority of viscous BUD is likely due to prolonged mucosal medication contact time, which was measured by scintigraphy.

\section{Side Effects of tCS}

Topical steroid therapy is believed to be safe. The most recognized side effect is Candida esophagitis in 5-30\% of patients $[3,5-7,21,22]$. Candida esophagitis was diagnosed in the majority of cases when performing follow-up endoscopies, meaning Candida infection did not evoke symptoms or swallowing discomfort. Approximately $1 \%$ of patients treated with tCS develop oral candidiasis regardless of whether BUD or FLU was administered and no matter what formulation and dose. Rinsing the mouth after taking the medication could not prevent the development oral candidiasis. To date, no evidence has been seen concerning adrenal suppression in up to 2 months of treatment, especially in children [16]. Another major issue of long-term treatment of EoE with $\mathrm{tCS}$ is atrophy of the esophageal mucosa. However, Straumann et al. [23] could not see a difference in the thickness of the esophageal squamous epithelium using histomorphometry in EoE patients versus healthy controls after 50 weeks of treatment with BUD.

\section{How Long Should We Treat EoE?}

There is only one prospective study describing the natural history of EoE following treatment [24]. After a 6-week treatment with FLU, 91\% of patients reported recurrent symptoms for a mean of 8.8 months after therapy was discontinued. Further, $69 \%$ of the patients repeated at least one second treatment course. One of the first treatment studies of systemic CS in pediatric EoE reported 90\% recurrence in symptoms after cessation of therapy [19]. Learning from these observations, it seems that EoE is a chronic remitting disorder requiring maintenance treatment, particularly with regard to minimizing esophageal remodeling.

In a randomized double-blind and placebo-controlled 50 -week trial with nebulized swallowed BUD $(0.25 \mathrm{mg}$ twice daily) in adult EoE patients, BUD was superior in maintaining clinical and histological remission in comparison to placebo [23]. No serious adverse events occurred in the BUD group and no atrophy of the esophageal epithelium was observed.

\section{Conclusion}

EoE is a chronic remitting disease of the esophagus which when untreated tends to lead to remodeling/fibrosis, resulting in complications such as stenosis and longlasting food impaction requiring endoscopic removal procedures. tCS are efficient in inducing symptomatic and histological remission with very low side effects - even over a long-term period. An optimized formulation (efficient drug delivery to the esophageal mucosa, easily applicable and integrated into daily life) of tCS remains to be developed and evaluated in further studies. A high relapse rate after short-term therapy has been observed, implying the need for long-term maintenance treatment. The duration of therapy and minimal dosage of tCS to keep EoE in remission have not been well defined and need further investigation and studies.

\section{Disclosure Statement}

The authors declare that no financial or other conflict of interest exists in relation to the content of the article. 


\section{References}

1 Liacouras CA, Furuta GT, Hirano I, et al: Eosinophilic esophagitis: updated consensus recommendations for children and adults. J Allergy Clin Immunol 2011;128:3-20.e6, quiz 21-22.

$\checkmark 2$ Arora AS, Yamazaki K: Eosinophilic esophagitis: asthma of the esophagus? Clin Gastroenterol Hepatol 2004;2:523-530.

$\checkmark 3$ Straumann A, Conus S, Degen L, et al: Budesonide is effective in adolescent and adult patients with active eosinophilic esophagitis. Gastroenterology 2010;139: 15261537.

$>4$ Konikoff MR, Noel RJ, Blanchard C, et al: A randomized, double-blind, placebo-controlled trial of fluticasone propionate for pediatric eosinophilic esophagitis. Gastroenterology 2006;131:1381-1391.

$\checkmark 5$ Dohil R, Newbury R, Fox L, et al: Oral viscous budesonide is effective in children with eosinophilic esophagitis in a randomized, placebocontrolled trial. Gastroenterology 2010;139: 418-429.

-6 Alexander JA, Jung KW, Arora AS, et al: Swallowed fluticasone improves histologic but not symptomatic response of adults with eosinophilic esophagitis. Clin Gastroenterol Hepatol 2012;10:742-749.

$>7$ Schaefer ET, Fitzgerald JF, Molleston JP, et al: Comparison of oral prednisone and topical fluticasone in the treatment of eosinophilic esophagitis: a randomized trial in children. Clin Gastroenterol Hepatol 2008;6:165-173.

8 Peterson KA, Thomas KL, Hilden K, et al: Comparison of esomeprazole to aerosolized, swallowed fluticasone for eosinophilic esophagitis. Dig Dis Sci 2010;55:1313-1319.
-9 Dellon ES, Sheikh A, Speck O, et al: Viscous topical is more effective than nebulized steroid therapy for patients with eosinophilic esophagitis. Gastroenterology 2012;143:321324.

10 Taft TH, Kern E, Keefer L, et al: Qualitative assessment of patient-reported outcomes in adults with eosinophilic esophagitis. J Clin Gastroenterol 2011;45:769-774.

11 Franciosi JP, Hommel KA, Bendo CB, et al: PedsQL eosinophilic esophagitis module: feasibility, reliability, and validity. J Pediatr Gastroenterol Nutr 2013;57:57-66.

12 Croese J, Fairley SK, Masson JW, et al: Clinical and endoscopic features of eosinophilic esophagitis in adults. Gastrointest Endosc 2003;58: 516-522.

13 Kerlin P, Jones D, Remedios M, Campbell C: Prevalence of eosinophilic esophagitis in adults with food bolus obstruction of the esophagus. J Clin Gastroenterol 2007;41:356361.

14 Desai TK, Stecevic V, Chang C, et al: Association of eosinophilic inflammation with esophageal food impaction in adults. Gastrointest Endosc 2005;61:795-801.

15 Straumann A, Bussmann C, Zuber M, et al: Eosinophilic esophagitis: analysis of food im paction and perforation in 251 adolescent and adult patients. Clin Gastroenterol Hepatol 2008;6:598-600.

16 Aceves SS, Newbury RO, Dohil R, et al: Esophageal remodeling in pediatric eosinophilic esophagitis. J Allergy Clin Immunol 2007;119:206-212.
17 Mishra A, Wang M, Pemmaraju VR, et al: Esophageal remodeling develops as a consequence of tissue specific IL-5-induced eosinophilia. Gastroenterology 2008;134:204-214.

18 Aceves SS, Newbury RO, Chen D, et al: Resolution of remodeling in eosinophilic esophagitis correlates with epithelial response to topical corticosteroids. Allergy 2010;65:109-116.

19 Liacouras CA, Wenner WJ, Brown K, Ruchelli E: Primary eosinophilic esophagitis in children: successful treatment with oral corticosteroids. J Pediatr Gastroenterol Nutr 1998; 26:380-385.

20 Aceves SS, Bastian JF, Newbury RO, Dohil R Oral viscous budesonide: a potential new therapy for eosinophilic esophagitis in children. Am J Gastroenterol 2007;102:22712279.

21 Aceves SS, Dohil R, Newbury RO, Bastian JF: Topical viscous budesonide suspension for treatment of eosinophilic esophagitis. J Allergy Clin Immunol 2005; 116:705-706.

22 Remedios M, Campbell C, Jones DM, Kerlin P: Eosinophilic esophagitis in adults: clinical, endoscopic, histologic findings, and response to treatment with fluticasone propionate. Gastrointest Endosc 2006;63:3-12.

23 Straumann A, Conus S, Degen L, et al: Longterm budesonide maintenance treatment is partially effective for patients with eosinophilic esophagitis. Clin Gastroenterol Hepatol 2011;9:400-409.

24 Helou EF, Simonson J, Arora AS: 3-yr-followup of topical corticosteroid treatment for eosinophilic esophagitis in adults. Am J Gastroenterol 2008;103:2194-2199. 